\title{
Combinatorial Adventures in Analysis, Algebra, and Topology
}

\section{Stephen Melczer, Marni Mishna, and Robin Pemantle}

The authors of this piece are organizers of the AMS 2020 Mathematics Research Communities summer conference Combinatorial Applications of Computational Geometry and Algebraic Topology, one of five topical research conferences offered this year that are focused on collaborative research and professional development for early-career

mathematicians. Researchers with experience in one or more of the areas of combinatorics, computational algebra, analysis, algebraic topology, and singularity theory are welcomed. Additional information can be

found at https: //www . ams . org/programs

/research-communities/2020MRC-CompGeom. Applications are open until February 15, 2020.

Capturing large-scale behavior of combinatorial objects can be difficult but very revealing. Consider Figure 1, for instance, which illustrates the behavior of random objects of large size in two combinatorial classes inspired by quantum computing and statistical mechanics.

Stephen Melczer is a CRM-ISM postdoctoral fellow at the Université du Québec à Montréal. His email address is sme1czer@sas. upenn. edu.

Marni Mishna is a professor of mathematics at Simon Fraser University. Her email address is mmi shna@sfu.ca.

Robin Pemantle is the Merriam Term Professor of Mathematics at the University of Pennsylvania. His email address is pemant 1 e@math. upenn.edu.

For permission to reprint this article, please contact:

reprint-permission@ams.org.

DOI: https://doi.org/10.1090/noti2031
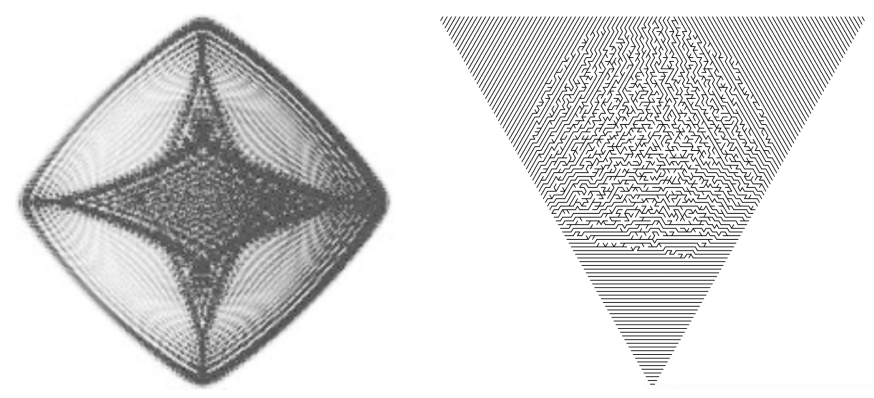

Figure 1. ACSV has been used to determine the shape of large random quantum random walks [3] (left) and cube groves [5] (right).

Perhaps surprisingly, complex analysis, algebraic geometry, topology, and computer algebra are important ingredients in such results, combining to form the relatively new field of analytic combinatorics in several variables (ACSV). ACSV builds on classic generating function techniques in combinatorics to provide new tools for the study of sequences and discrete objects. Applications previously studied in the literature include queuing systems, bioinformatics, data structures, random tilings, special functions, and integrable systems. Ongoing research efforts from the combinatorial side concern an evolving study of lattice paths [8] and objects from representation theory and algebraic combinatorics [14].

The purpose of this note is to give a brief overview of ACSV and its history to drive interest among those in any of the diverse areas touched by the topic. A large collection of worked examples can be found in [15] and a detailed treatment of ACSV in [16]. 


\section{Analytic Combinatorics}

The use of analytic techniques in combinatorics and probability theory has a long history, dating back to the eighteenth-century work of Laplace [12] and contemporaries such as Stirling [18] and de Moivre [9]. In modern times, the use of real and complex analysis to derive asymptotic behavior is the domain of analytic combinatorics [11], a field which finds application in many areas of mathematics, computer science, and the natural sciences. The key idea behind such results is that important properties of a sequence $\left(f_{n}\right)=\left(f_{0}, f_{1}, \ldots\right)$ of real or complex numbers are easily deduced from the generating function

$$
F(z)=\sum_{n \geq 0} f_{n} z^{n}=f_{0}+f_{1} z+\cdots .
$$

Although much can be deduced treating $F$ only as a formal series [20], when $f_{n}$ grows at most exponentially as $n$ goes to infinity then $F$ defines a complex-analytic function at the origin. The powerful methods of complex analysis can then be used to study properties of $f_{n}$, including its asymptotic behavior.

Indeed, the Cauchy integral formula implies that for every natural number $n$ the coefficient $f_{n}$ can be represented by a contour integral

$$
f_{n}=\frac{1}{2 \pi i} \int_{\mathcal{C}} F(z) z^{-n-1} d z,
$$

where $\mathcal{C}$ is a positively oriented circle sufficiently close to the origin. The singularities of the generating function play a crucial role in determining sequence asymptotics: the domain of integration $\mathcal{C}$ can be deformed without changing the value of the Cauchy integral as long it does not cross any singularities of $F$. Setting some technicalities aside, each singularity of $F$ gives a "contribution" to asymptotics of $f_{n}$ depending on its location in the complex plane and the local behavior of $F$ near the singularity. This approach is powerful enough to be completely automated for large classes of generating functions, such as rational and algebraic functions, and is flexible enough to extend as required by applications. A thorough development of this univariate theory and a survey of its applications can be found in the fantastic text of Flajolet and Sedgewick [11].

\section{ACSV}

In contrast to the well-developed univariate theory, until recently there was no general framework for the study of multivariate generating functions. To rectify this gap in the literature, over the last two decades the theory of ACSV has been developed [16]. Here we focus on a rational function $F(\mathbf{z})=F\left(z_{1}, \ldots, z_{d}\right)$ in $d>1$ variables with power series expansion

$$
F(\mathbf{z})=\sum_{\mathbf{i} \in \mathbb{N}^{d}} f_{\mathbf{i}} \mathbf{z}^{\mathbf{i}}=\sum_{\mathbf{i} \in \mathbb{N} d} f_{i_{1}, \ldots, i_{d}} z_{1}^{i_{1}} \cdots z_{d}^{i_{d}} .
$$

Given $\mathbf{r} \in \mathbb{N}^{d}$, the $\mathbf{r}$-diagonal of $F(\mathbf{z})$ is the sequence $\left(f_{n \mathbf{r}}\right)$. Many mathematical sequences arise naturally as diagonals of explicit rational functions. Furthermore, coefficient series for all algebraic functions may be embedded as generalized diagonals of rational functions [17]. It is conjectured that the same is true of all globally bounded D-finite functions [7, Conjecture 4].

Example 1. A key step in Apéry's proof [19] of the irrationality of $\zeta(3)$ is determining asymptotics of the sequence

$$
b_{n}=\sum_{k=0}^{n}\left(\begin{array}{l}
n \\
k
\end{array}\right)^{2}\left(\begin{array}{c}
n+k \\
k
\end{array}\right)^{2} .
$$

Because it is a sum of nonnegative terms, saddle point approximations will produce the desired asymptotics.

Such sums with mixed signs are notoriously difficult to estimate. However, any binomial sum sequence regardless of signs can automatically be written as the $\mathbf{1}$-diagonal of a rational function [6]. For instance, Apéry's sum is the 1-diagonal of the four-variable rational function

$$
\frac{1}{1-t(1+x)(1+y)(1+z)(1+y+z+y z+x y z)} \text {. }
$$

The methods of ACSV determine asymptotics of $b_{n}$ completely automatically [13].

Although the $\mathbf{r}$-diagonal of a fixed rational function is defined only when $\mathbf{r}$ has integer coordinates, ACSV shows that asymptotics along most ${ }^{1}$ directions $\mathbf{r} \in \mathbb{R}_{>0}^{d}$ can be well defined by a limiting procedure.

\section{Multivariate Analysis and Singularity Theory}

As in the univariate setting, the methods of ACSV start from a Cauchy integral formula

$$
f_{n \mathbf{r}}=\frac{1}{(2 \pi i)^{d}} \int_{\mathcal{C}} F(\mathbf{z}) \mathbf{z}^{-n \mathbf{r}-\mathbf{1}} d z_{1} \cdots d z_{d},
$$

where $\mathcal{C}$ is now a product of positively oriented circles sufficiently close to the origin. The singularities of $F(\mathbf{z})$ again play a crucial role in asymptotics. If $F(\mathbf{z})=G(\mathbf{z}) / H(\mathbf{z})$ is a rational function with coprime numerator $G$ and denominator $H$, then the singularities of $F$ form the set $\mathcal{V}=\{\mathbf{z} \in$ $\left.\mathbb{C}^{d}: H(\mathbf{z})=0\right\}$.

Once the dimension is greater than 1 , the singular set $\mathcal{V}$ is no longer discrete but is an algebraic variety of positive dimension. In the geometrically simplest case, when $\mathcal{V}$

\footnotetext{
${ }^{1}$ Asymptotics of the $\mathbf{r}$-diagonal vary smoothly with $\mathbf{r}$ inside open cones of $\mathbb{R}_{>0}^{d}$ The boundaries of these cones form $(d-1)$-dimensional sets where asymptotics can sharply change.
} 
forms a smooth manifold, one searches for a (generically finite) set of critical points near which the Cauchy integral can be approximated by saddle-point integrals which are easy to estimate asymptotically. When some of these critical points lie on the boundary of the generating function domain of convergence and other mild conditions hold, asymptotics can be computed using explicit formulas. In the absence of critical points on the boundary of convergence or when the geometry of this domain is difficult to establish, topological techniques may be applied to determine which critical points are responsible for the coefficient asymptotics. This was carried out in two dimensions in [10]; generalizing to higher dimensions is an open problem.

Without restricting the geometry of $\mathcal{V}$, recent work [4] has shown how stratified Morse theory helps determine the deformations of $\mathcal{C}$ in $\mathbb{C}^{d} \backslash \mathcal{V}$ which yield integrals of the type analyzed in classic works on singularity theory $[1,2]$. The techniques of ACSV thus rely on an interesting mix of computer algebra, singularity theory, algebra, geometry, and topology. By asking different questions, ACSV promotes new work in these areas. For example, any advances in the automatic computation of singular integrals can be applied back to the wavelike partial differential equations for which purpose singularity theory was developed in the 1970 s and 1980s. Indeed this development may already be ripe for a resurgence as it couples with modern computer algebra methods.

\section{AMS Math Research Community}

Being a new and rapidly growing field which incorporates techniques from diverse corners of mathematics, ACSV has a rich collection of problems accessible to early researchers in many areas. Some of these problems are computational in nature, including the development of computer algebra tools for asymptotics under varying assumptions. Others are topological at heart, such as computing intersection and linking numbers of attachment cycles which arise in the Morse theoretic constructions discussed above. Many relate to asymptotic computations of saddle-point asymptotics in degenerate settings which appear in combinatorial applications. Of course, after developing such tools there is also a need for combinatorialists to apply them to problems of differing scope. Most of the advances in ACSV have come from combinatorial problems just beyond the reach of existing techniques.

We thus encourage early-career mathematicians with experience in any one of the areas of combinatorics, computational algebra, analysis, algebraic topology, singularity theory, or hyperbolic partial differential equations to apply to our upcoming Math Research Community. Our hope is to have participants from a wide variety of fields interacting with each other to develop this exciting area of mathematics.

\section{References}

[1] Arnol'd VI, Guseĭn-Zade SM, Varchenko AN. Singularities of Differentiable Maps. Vol. II, Monodromy and asymptotics of integrals, translated from the Russian by Hugh Porteous, translation revised by the authors and James Montaldi, Monographs in Mathematics, vol. 83, Birkhäuser Boston, Inc., Boston, MA, 1988. MR966191

[2] Atiyah MF, Bott R, Gärding L. Lacunas for hyperbolic differential operators with constant coefficients. I, Acta Math. (124):109-189, 1970, DOI 10.1007/BF02394570 MR470499

[3] Baryshnikov Y, Brady W, Bressler A, Pemantle R. Two-dimensional quantum random walk, J. Stat. Phys., no. 1 (142):78-107, 2011, DOI 10.1007/s10955-010-00982. MR2749710

[4] Baryshnikov Y, Melczer S, Pemantle R. Critical points at infinity for analytic combinatorics, Submitted, arxiv . org/abs/1905.05250, 2019.

[5] Baryshnikov Y, Pemantle R. Asymptotics of multivariate sequences, part III: Quadratic points, Adv. Math., no. 6 (228):3127-3206, 2011, DOI 10.1016/i.aim.2011.08.004 MR2844940

[6] Bostan A, Lairez P, Salvy B. Multiple binomial sums, J. Symbolic Comput., part 2 (80):351-386, 2017, DOI 10.1016/j.jsc.2016.04.002. MR3574517

[7] Christol G. Globally bounded solutions of differential equations, Analytic Number Theory (Tokyo, 1988), Springer, Berlin, 1990, 45-64, DOI 10.1007/BFb0097124 MR1071744

[8] Courtiel J, Melczer S, Mishna M, Raschel K. Weighted lattice walks and universality classes, J. Combin. Theory Ser. A (152):255-302, 2017, DOI 10.1016/j.jcta.2017.06.008 MR3682734

[9] de Moivre A. Miscellanea analytica de seriebus et quadraturis, J. Tomson and J. Watts, London, 1730.

[10] DeVries T, van der Hoeven J, Pemantle R. Effective asymptotics for smooth bivariate generating functions, $\mathrm{On}$ line J. Anal. Comb. (7):24, 2012.

[11] Flajolet P, Sedgewick R. Analytic Combinatorics, Cambridge University Press, Cambridge, 2009. MR2483235

[12] Laplace P-S. Mémoire sur la probabilité des causes par les évènemens, Mémoires de mathématique et de physique (Tome VI):621-656, 1774. gal1ica.bnf.fr /ark:/12148/bpt6k3482c/f685

[13] Melczer S, Salvy B. Effective coefficient asymptotics of multivariate rational functions via semi-numerical algorithms for polynomial systems, arxiv . org 2019.

[14] Mishna M, Rosas M, Sundaram S. An elementary approach to the quasipolynomiality of the kronecker coefficients, https: //arxiv .org/abs/1811. 10015, 2018.

[15] Pemantle R, Wilson MC. Twenty combinatorial examples of asymptotics derived from multivariate generating functions, SIAM Rev., no. 2 (50):199-272, 2008, DOI $10.1137 / 050643866$ MR2403050 
[16] Pemantle R, Wilson MC. Analytic Combinatorics in Several Variables, Cambridge Studies in Advanced Mathematics, vol. 140, Cambridge University Press, Cambridge, 2013. MR3088495

[17] Safonov KV. On power series of algebraic and rational functions in $\mathbf{C}^{n}$, J. Math. Anal. Appl., no. 2 (243):261-277, 2000, DOI 10.1006/jmaa.1999.6667 MR1741523

[18] Stirling J. Methodus differentialis: sive tractatus de summatione et interpolatione serierum infinitarium, London, 1730.

[19] van der Poorten A. A proof that Euler missed...Apéry's proof of the irrationality of $\zeta(3)$, An informal report, Math. Intelligencer, no. 4 (1):195-203, 1978/79, DOI 10.1007/BF03028234 MR547748

[20] Wilf HS. generatingfunctionology, 3rd ed., A K Peters, Ltd., Wellesley, MA, 2006. MR2172781

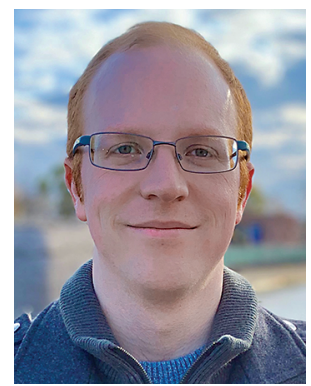

Stephen Melczer

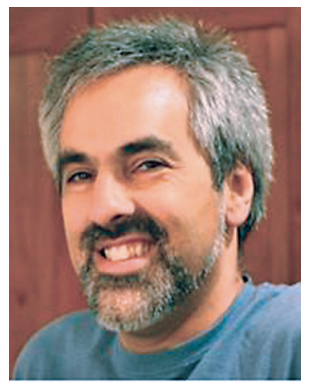

Robin Pemantle

\section{Credits}

Figure 1 and photo of Robin Pemantle are courtesy of Robin Pemantle.

Photo of Stephen Melczer was taken by Celia Laur.

Photo of Marni Mishna is courtesy of Marni Mishna.
AMS

AUTHOR

RESOURCE

CENTER

The Author Resource Center is a collection of information and tools available to assist you to successfully write, edit, illustrate, and publish your mathematical works.

\section{To begin utilizing these important resources, visit:}
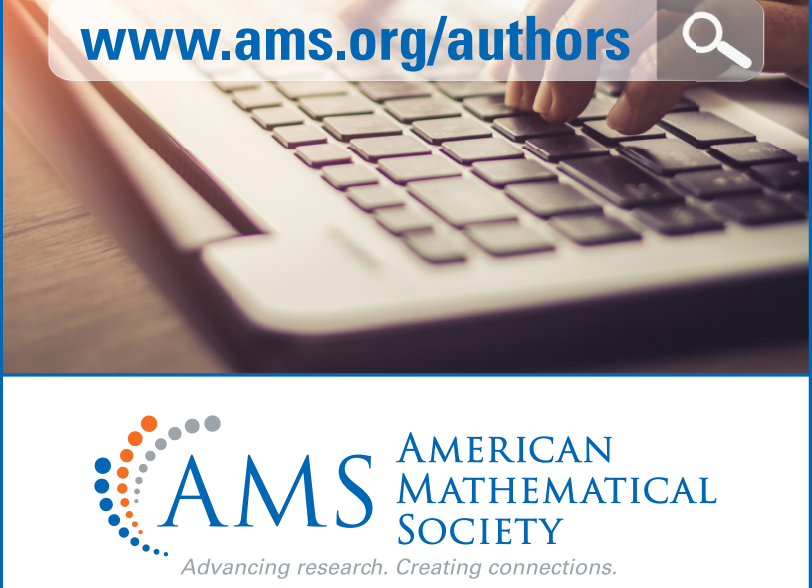